Abstract Silicon microring resonators very often exhibit resonance splitting due to backscattering. This effect is hard to model in a quantitative and predictive way. This paper presents a behavioral circuit model for ring resonators that quantitatively explains the wide variations in resonance splitting observed in experiments. The model is based on an in-depth analysis of the contributions to backscattering by both the ring waveguides and the coupling sections, and it accurately explains the origin of asymmetric resonance splitting. Backscattering transforms unidirectional ring resonators into bidirectional circuits by coupling the clockwise and counter-clockwise circulating modes.In high$Q$ rings this will induce visible resonance splitting, but due to the stochastic nature of backscattering this splitting is different for each resonance. Our model, based on temporal coupled mode theory, and the associated fitting method are both accurate and robust, and can also, for the first time, explain asymmetrically split resonances. The cause of asymmetric resonance splitting is identified as the backcoupling in the coupling sections. This is experimentally confirmed, and we further analyze the dependency on gap and coupling length. Moreover, the wide variations in resonance splitting of one spectrum is also analyzed and successfully explained by our circuit model that incorporates most linear parasitic effects in the ring resonator. This analysis uncovers multi-cavity interference within the ring as the source of this variation.

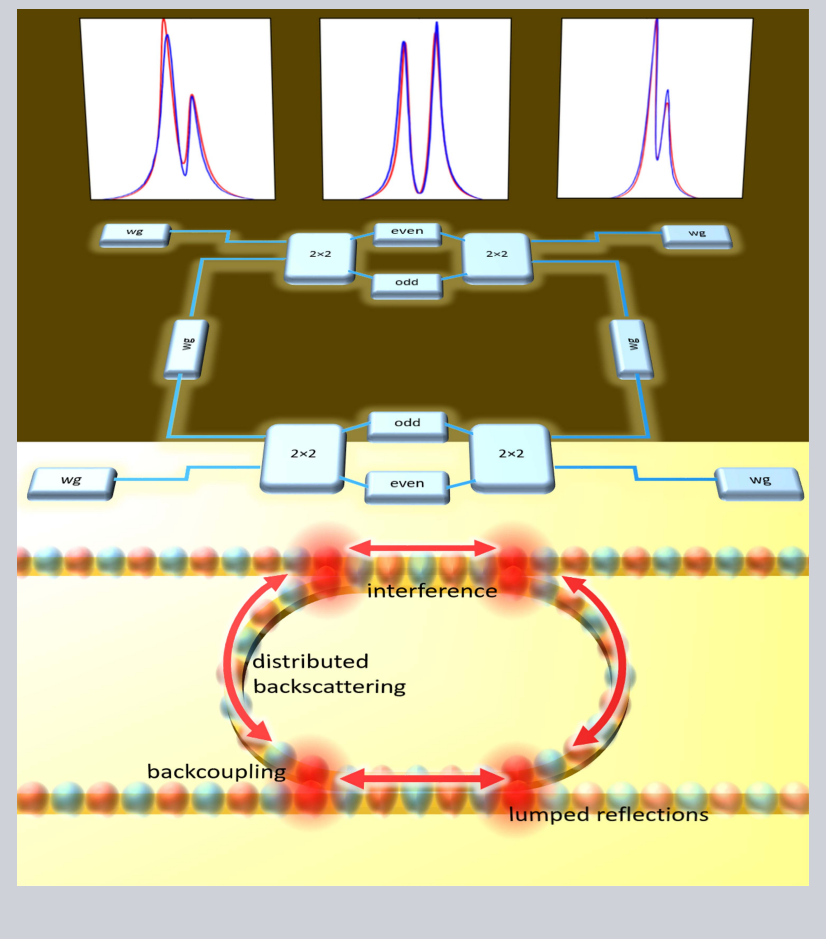

\title{
Backscattering in Silicon Microring Resonators: A Quantitative Analysis
}

\author{
Ang $\mathrm{Li}^{1,2}$, Thomas Van Vaerenbergh ${ }^{1,2,3}$, Peter De Heyn ${ }^{3}$,Peter Bienstman ${ }^{1,2}$, and Wim \\ Bogaerts ${ }^{1,2,4}$
}

\section{Introduction}

Silicon microring resonators are extremely useful devices to create compact WDM filters, (bio)sensors, all-optical signal processing, optical switches, optical wavelength converters, microwave photonics, and laser cavities [1-7]. The use of silicon gives a high refractive-index contrast, allowing for compact rings with a large free spectral range (FSR). In addition, the material system is compatible with CMOS manufacturing processes, offering a route towards largescale integration and mass-manufacturing.

The basic operation principles of (silicon) microring resonators have already been described extensively $[8,9]$. Basically, an ideal add-drop ring filter operates as shown in Fig. 1a. Light coupled from the in port is coupled to the ring waveguide and circulates clockwise (CW) in one direction to be extracted at the drop port when the wavelength is near the resonance wavelength, or coupled back to the pass port for other wavelengths. The transmission spectra at the drop and pass port consist of a series of Lorentzian-shaped resonances, with no light coming out the add port and in port, as is evident from Fig. 2a. In this unidirectional behavior the counterclockwise (CCW) mode is never excited.

However, any nonideality in the ring waveguide can lead to small backreflections that can excite the CCW mode. Especially near resonance, when the optical intensity in the ring waveguide is high, backscattering can lead to a coherent build-up of this reflected light, even if the individual reflections are very weak. This excitation of the $\mathrm{CCW}$ mode results in undesired power output at the $a d d$ and in port, and a perturbation of the ideal single resonance state. The backreflection couples the degenerate $\mathrm{CW}$ and the $\mathrm{CCW}$ mode into

\footnotetext{
${ }^{1}$ Photonics Research Group, Ghent University-IMEC, Ghent,9000 Belgium.

${ }^{2}$ Center for Nano- and Biophotonics (NB-photonics), Ghent University, Belgium

${ }^{3}$ Hewlett Packards Labs, 1501 Page Mill Road, Palo Alto, CA 94304, USA.

${ }^{4}$ IMEC, Kapeldreef 75, Leuven, Belgium.

${ }^{5}$ Luceda Photonics, Dendermonde, Belgium.

Corresponding author: e-mail: ang.li@intec.ugent.be
} 


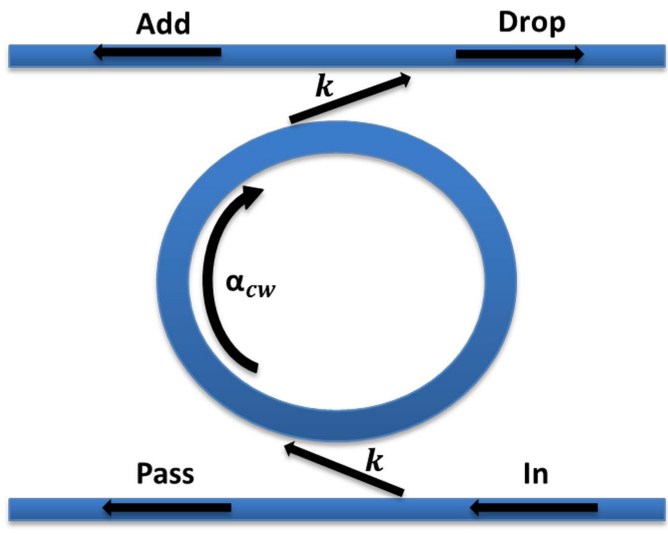

(a)

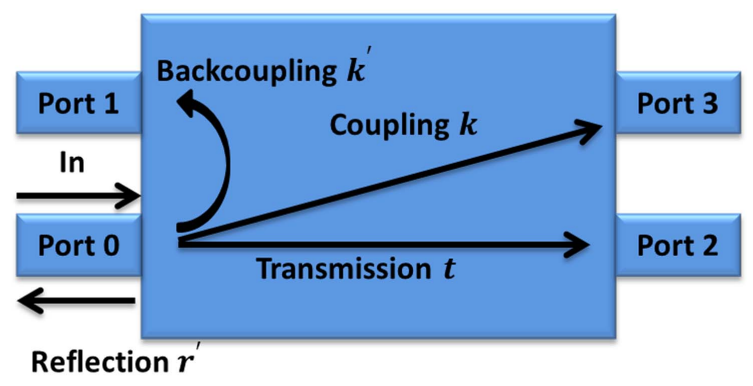

(c)

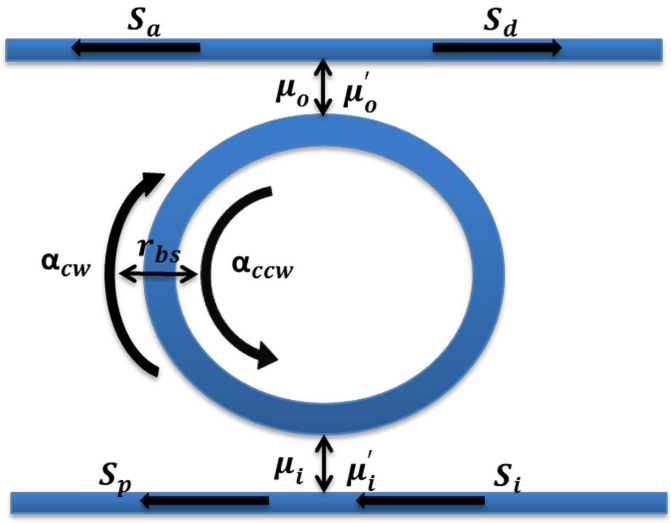

(b)

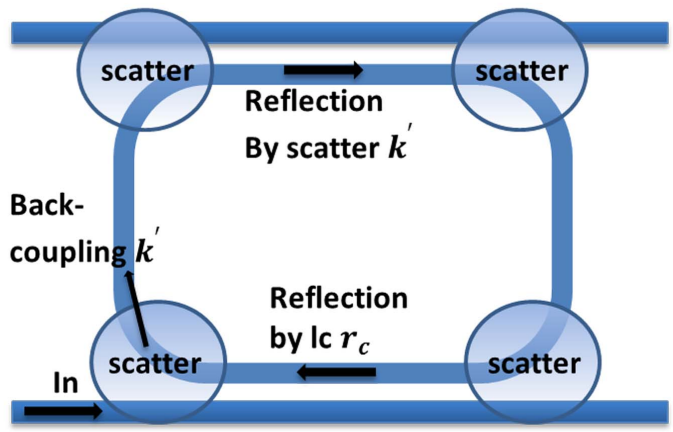

$(\boldsymbol{d})$

Figure 1: Ring resonator models for backscattering. (a) Schematic of an ideal ring resonator without backscattering. Light coupled from the inport will circulate in the ring waveguide and extracted to the drop port near the resonance wavelength, or continue to the pass port for other wavelengths. (b) t-CMT model for the ring circuit with backscattering. CCW and CW modes are degenerate in ring resonators without backscattering, and only one is excited by each input. However, due to backscattering $r_{b s}$, they are coupled with each other. The factors $\mu_{x}, \mu_{x}^{\prime}$ stand for the mutual coupling of the directional couplers. (c) A simplified schematic of a $2 \times 2$ directional coupler. Ideally, the backcoupling $k^{\prime}$ and reflection $r^{\prime}$ are zero. (d) Illustration of the extra reflections caused by directional couplers in a ring. The directional coupler in a ring resonator can be physically divided into three parts, two scattering centers at the beginning and end, and a distributed scatterer in the straight section due to extra coupling length. Scatterers will cause unwanted backcoupling to adjacent port as well as reflection to in port, while coupling length will bring roughness induced backscattering.

two new resonance states with a mixed CW/CCW nature, and different resonance wavelengths. In rings with a very high Q-factor, whose linewidth is sufficiently narrow, this will induce a visible peak splitting in the spectral response, and the ring characteristics can be seriously deteriorated compared to the ideal Lorentzian [10]. As the backreflection can have many contributions, it is also wavelength dependent, and therefore the resonance splitting can vary from one resonance peak to the next. This is shown in Fig. 2b, which is taken from a typical measurement of silicon microring resonators and Fig. 3, which shows the distributions of split ratios of 6 sets of ring resonators with 7 rings with variable lengths in each set. This resonance splitting will cause problems for many ring resonator applications:

- In ring-based sensors the shift of the resonance wavelength might not be correctly detected [11].
- In ring-resonator-based tunable lasers, the reflection at in port as well as the distortion of the ring's transmission spectrum may cause laser instabilities.

- In ring-resonator-assisted loss characterization techniques, wrong model parameters will be extracted from an imperfect fitting of split resonances [12].

- Ring-resonator-based wavelength-division multiplexing (WDM) filters will deviate from the designed filter specifications as the Q-factor cannot be well controlled.

- Ring-assisted microwave detection circuits could result in the wrong peak power extracted from a split resonance [6].

However, in some situations, the backscattering in ring resonators can be harnessed and provide attractive benefits: for instance, the extinction ratio of a filter can be strengthened [13].

In order to correctly deal with such split resonances, and fully avoid or take advantage of backscattering, an ac- 


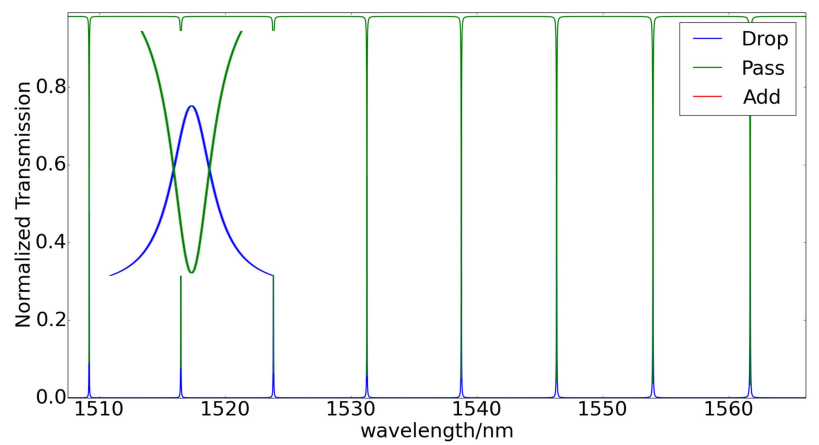

(a) A spectrum of an ideal ring resonator. Clean Lorentzian-shaped resonances at drop and pass ports, no light at in and add ports.

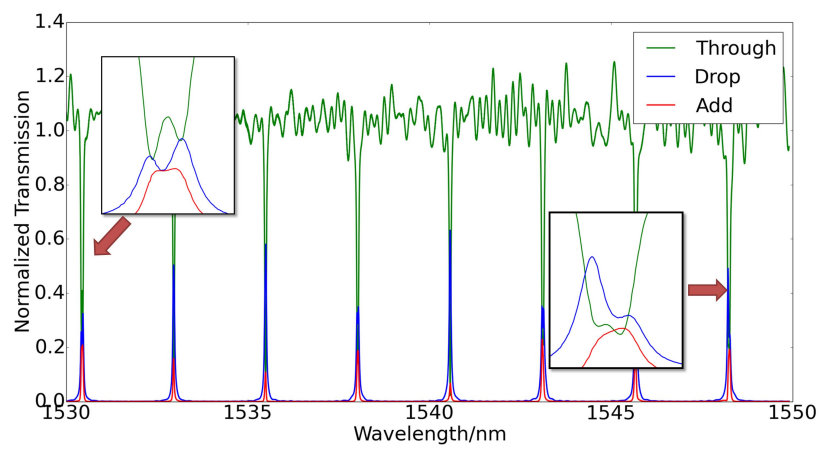

(b) A measured spectra of a silicon ring resonator. Resonances exhibit different shapes due to splitting.

Figure 2: Examples of spectra of ideal and real ring resonators, respectively.
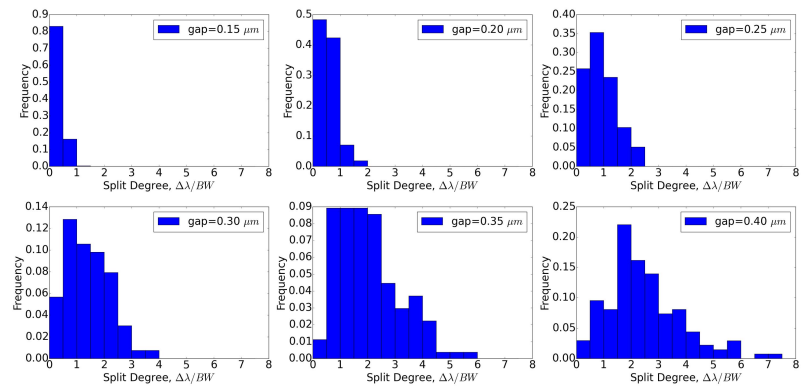

Figure 3: Histogram of split ratios of 6 sets of ring resonators; Each set contains 7 rings with variable lengths but the same coupling gap and coupling length (in total there are as many as 1080 resonances ). When a resonance split is larger than half of $3 \mathrm{~dB}$ bandwidth, it becomes visible.

curate and robust model of the ring resonator is required. With today's models, the fitting of split resonances does not correspond very well with the measured reality. Current fitting models typically describe only symmetrically split resonances [12-15], even though many resonances are asymmetrically split. No published models can explain this asymmetry in a satisfactory manner. Still, our experimental data shows that, in many cases, a significant fraction of the split resonances are asymmetrically split (see Fig. 4),

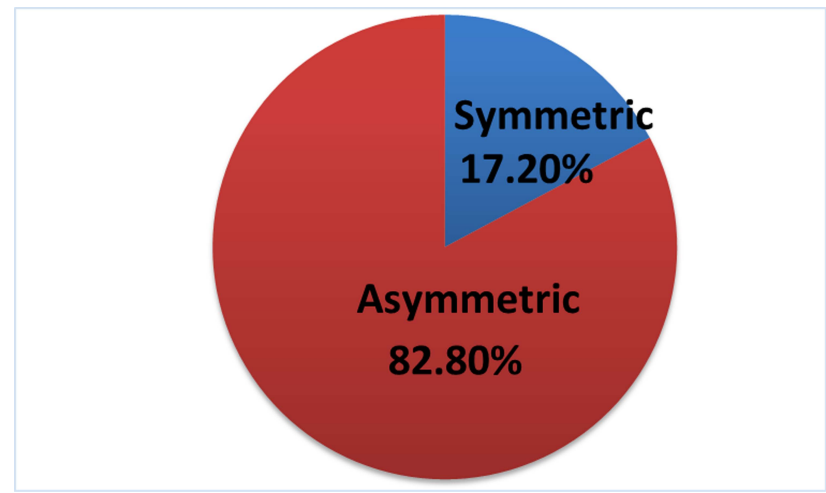

Figure 4: A pie chart clearly shows the dominance of asymmetric splitting among all of the splitting resonances we measured from 252 rings ( in total more than 550 resonances exhibit splitting ).

making it necessary to build a fitting model which could handle all kinds of resonances. Another difficulty is the experimental identification and quantification of the different contributions to the backscattering and peak splitting. So far, analysis of measurements on silicon microrings has attributed all backscattering to waveguide roughness, which is distributed along the ring $[10,16]$. The possible contribution to backreflection from the directional couplers has only been theoretically proposed [17], but has not yet been experimentally verified and quantitatively characterized. In this paper, we develop a fitting model that can explain and reproduce all kinds of split and non-split resonances, and identify the origin of asymmetrically split resonances. Moreover, we propose and experimentally prove that, besides waveguide roughness, the directional couplers indeed contribute to backscattering in silicon microrings. We also investigate how the coupler's gap as well as extra coupling length will influence coupler-induced backscattering.

In the following section, we introduce our theoretical analysis and the models for the individual contributions to the backscattering, as well as the model based on temporal coupled mode theory (t-CMT) for the complete ring circuit with backscattering, with which we can fit all resonances one by one in an automatic way. In section 3, we will then identify the origin of this asymmetry in most split resonances, namely the backcoupling of directional couplers, and prove that without this backcoupling we cannot explain all types of asymmetry. Subsequently, the fitting results for measured ring spectra and experimentally characterization of backscattering are presented in section 4. Also, the influence of coupler gap, ring length and coupling length on backscattering is shown. In the section after that, we will make our model more rigorous and complete by revealing the reason why individual resonances can be so significantly different within the same spectrum. In this section, we will improve our fitting model by fitting the circuit and its whole spectrum instead of fitting the resonances one by one. 


\section{2. $\mathrm{t}-\mathrm{CMT}$ model for a ring resonator}

Temporal Coupled-Mode-Theory (t-CMT) is a very good and useful model to analyse a single resonance of a ring resonator [18]. Our model improves upon existing models in that it includes both distributed and localized (lumped) backscattering: distributed backscattering is caused by waveguide sidewall roughness distributed along the ring circumference, while lumped backscattering is caused by localized discontinuities in the ring. In most rings, these discontinuities can be found in the coupling sections.

In this section, we first introduce our t-CMT model for the microring circuit with backscattering taken into consideration. After that, we discuss the models and analysis for the individual contributions to backscattering, namely the directional couplers (lumped) and waveguide roughness (distributed).

A perfect ring resonator supports two degenerate modes, a propagating clockwise $(\mathrm{CW})$ and counter-clockwise (CCW), respectively. Ideally, only one of them is excited by each input. However, due to backscattering in the ring waveguide, these two modes can become coupled with each other, thus excited simultaneously, as shown in Fig. 1b. When introducing a lumped reflector with field reflectivity $r_{b s}$ to represent backscattering, $\mathrm{t}-\mathrm{CMT}$ can also be used to analyse a non-ideal ring resonator.

Equations (1)-(4) are derived from our t-CMT model describing the two coupled resonance modes, and the transmission at drop port and $a d d$ port, respectively (the equations for the in and pass port are similar):

$$
\begin{gathered}
\frac{d \alpha_{c w}}{d t}=j\left(\omega_{0}+j \frac{1}{\tau_{t o t}}\right) \alpha_{c w}-j \mu_{12} \alpha_{c c w}-j \mu_{i} S_{i} \\
\frac{d \alpha_{c c w}}{d t}=j\left(\omega_{0}+j \frac{1}{\tau_{t o t}}\right) \alpha_{c c w}-j \mu_{21} \alpha_{c w}-j \mu_{i}^{\prime} S_{i} \\
S_{d}=-j \mu_{o} \alpha_{c w}-j \mu_{o}^{\prime} \alpha_{c c w} \\
S_{a}=-j \mu_{o}^{\prime} \alpha_{c w}-j \mu_{o} \alpha_{c c w}
\end{gathered}
$$

- $\alpha_{c c w}, \alpha_{c w}$ are the amplitudes of these two modes respectively [18].

- $\omega_{0}$ is the intrinsic resonant frequency of the ring, depending on the ring's physical parameters.

$-\frac{1}{\tau_{\text {tot }}}=\frac{1}{\tau_{l}}+\frac{1}{\tau_{i}}+\frac{1}{\tau_{o}}$, is the total decay rate of the ring circuit, including the intrinsic loss rate $\frac{1}{\tau_{l}}$, out- and incoupling rate $\frac{1}{\tau_{i}}$ and $\frac{1}{\tau_{o}}$. We can assume the last two are identical if the two couplers are designed to be identical, as is often the case. The relation between decay rate and mutual coupling is [18]:

$$
\mu_{x}^{2}=\frac{2}{\tau_{x}}
$$

- $\mu_{i}$ and $\mu_{o}$ are the mutual forward coupling of two directional couplers respectively, the dependency on power forward coupling coefficient in space $K=k^{2}$ is [18] :

$$
\mu_{i}^{2}=\mu_{o}^{2}=K_{i} \frac{v_{g}}{L}=K_{i} \frac{c}{n_{g} L}
$$

- $v_{g}$ is the group velocity in the ring circuit,

- $L$ is the physical length of the ring,

- $c$ is the light speed in vacuum,

- $n_{g}$ is the group index of the ring,

- $\mu_{12}, \mu_{21}$ refer to the mutual coupling of a lumped reflector inside the ring waveguide, which we use as a model for the backscattering. Based on the fact that the strength of backscattering should be independent of the propagation direction, we assume $\mu_{12}, \mu_{21}$ to have the same amplitudes. However, they might have different phase due to the stochastic and distributed nature of the backscattering which we capture in a lumped element: the effective coupling position for the two modes may be different, as also in-depth discussed In [19], where a circuit model for backscattering that takes both amplitude and phase stochastic feature into consideration, is proposed. The dependency on field reflectivity $r_{b s}$ is:

$$
\mu_{12}=r_{b s} \frac{v_{g}}{L}=r_{b s} \frac{c}{n_{g} L}
$$

Note that, even though in wavelength domain the field reflectivity $r_{b s}$ is also reported to show a stochastic nature $[16,19]$, while in our model, the $r_{b s}$ within one resonance is considered to be constant, due to the ultra narrow bandwidth of a ring resonance, which is measured to be around $10-40 \mathrm{pm}$.

- $\mu_{i}^{\prime}$ and $\mu_{o}^{\prime}$ are related to the backcoupling of the coupler. Similarly to $\mu_{i}$, the dependency on power backcoupling coefficient $K^{\prime}=\mathbf{k}^{\prime 2}$ is :

$$
\mu_{i}^{\prime 2}=\mu_{o}^{\prime 2}=K^{\prime} \frac{v_{g}}{L}=K^{\prime} \frac{c}{n_{g} L} .
$$

For simplicity, we introduce a dimensionless factor $f$ to mathematically describe the backcoupling $k^{\prime}$ relative to the forward coupling coefficient $k$ :

$$
k^{\prime}=f k, \mu_{i}^{\prime}=f \mu_{i}
$$

Note that $f$ can be complex, so there can be a phase difference between forward coupling and backcoupling.

- $S_{x}$ refers to the amplitude of the wave at each port. It is normalized such that $S_{x}^{2}$ has the unit of power.

After solving equations (3) and (4), we get the amplitudes at the drop port $S_{d}$ and add port $S_{a}$ as equations (10) and (12). For comparison, the case without any backscattering is given as equation (11) and (13):

$$
\begin{gathered}
\left.\frac{S_{d}}{S_{i}}\right|_{b s}=\frac{A_{d}}{2}\left[\frac{\frac{B W_{0}}{2}(1-f)^{2}}{j\left(\omega-\omega_{1}\right)+\frac{B W_{1}}{2}}+\frac{\frac{B W_{0}}{2}(1+f)^{2}}{j\left(\omega-\omega_{2}\right)+\frac{B W_{2}}{2}}\right] \\
\left.\frac{S_{d}}{S_{i}}\right|_{\text {ideal }}=A_{0}\left[\frac{\frac{B W_{0}}{2}}{j\left(\omega-\omega_{0}\right)+\frac{B W_{0}}{2}}\right]
\end{gathered}
$$




$$
\begin{gathered}
\left.\frac{S_{a}}{S_{i}}\right|_{b s}=\frac{A_{a}}{2}\left[-\frac{\frac{B W_{0}}{2}(1-f)^{2}}{j\left(\omega-\omega_{1}\right)+\frac{B W_{1}}{2}}+\frac{\frac{B W_{0}}{2}(1+f)^{2}}{j\left(\omega-\omega_{2}\right)+\frac{B W_{2}}{2}}\right] \\
\left.\frac{S_{a}}{S_{i}}\right|_{\text {ideal }}=0
\end{gathered}
$$

- $A_{x}$ is an dimensionless factor, scaled by potential transmission losses in the circuit. For ideal circuits, where no extra loss is present, $A_{X}=1$.

- $B W_{0}$ is the $3 \mathrm{~dB}$ frequency bandwidth of the ring when there is no backscattering at all, i.e. an ideal ring.

- $\left(\omega_{1}, B W_{1}\right)$ and $\left(\omega_{2}, B W_{2}\right)$ are the (central frequencies, frequency bandwidths) of the $\mathrm{CW}$ resonance mode $\left(\alpha_{c w}\right)$ and CCW mode $\left(\alpha_{c c w}\right)$ respectively.

$$
\begin{aligned}
\omega_{1} & =\omega_{0}+\mu_{0} \cos \frac{\phi_{\mu}}{2} \\
\omega_{2} & =\omega_{0}-\mu_{0} \cos \frac{\phi_{\mu}}{2} \\
B W_{1} & =B W_{0}+2 \mu_{0} \sin \frac{\phi_{\mu}}{2} \\
B W_{2} & =B W_{0}-2 \mu_{0} \sin \frac{\phi_{\mu}}{2}
\end{aligned}
$$

with $\mu_{0}=\left|\mu_{12} \mu_{21}\right|, \phi_{\mu}=\angle \mu_{12} \mu_{21} . \phi_{\mu}$ is dependent on the effective coupling position between the two circulating modes, and our fitting results shows that $\phi_{\mu}$ is close to 0 , this means that $\phi_{\mu_{12}}=-\phi_{\mu_{21}}$, in other words, the positions where coupling between these two modes effectively happen, are the same. What's more, this result also reveals that it's actually a conservative coupling instead of a dissipative coupling between these two modes.

From equations (14)-(17), we observe the distortion of the electric field at the drop port and the emergence of light at the $a d d$ port, due to $\mu_{12}$ and $f$. Instead of a single resonance with a Lorentzian line shape, there are now two resonances with their own resonance frequency/wavelength, bandwidth and peak power. When the separation between the two resonance frequencies becomes sufficiently large compared to their bandwidth, a visible peak splitting can be observed.

Obviously, $\mu_{12}$, i.e. backscattering in the ring, is responsible for the separation of the resonance frequencies, which is in agreement with models formerly published in literature [20]. In terms of the relative power in these two modes, there seems to be a dependence on both the bandwidth and the backcoupling factor $f$. The existence of backcoupling can thus be deduced from experimental data. Table 1 shows the characteristics of different split resonances from the same ring resonator. If there were no backcoupling at all $(f=0)$, the only reason that there could be an asymmetry in the peak power of the two modes is that their bandwidths are different, as can be seen in the denominator of equation (10). As a consequence, a larger bandwidth will lead to a lower peak power, and a large difference between peak

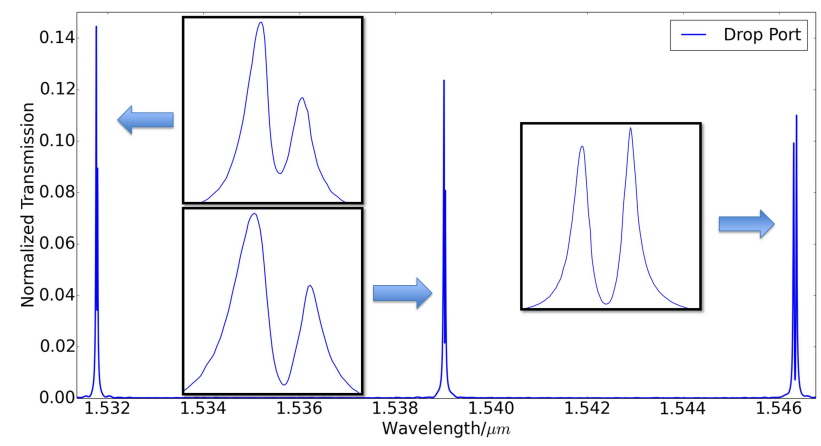

Figure 5: A measured spectrum containing 3 split resonances.

\begin{tabular}{|c|c|c|c|}
\hline & Resonance 1 & Resonance 2 & Resonance 3 \\
\hline$B W_{1} / p m$ & 16.7188 & 14.1098 & 21.0971 \\
\hline$B W_{2} / p m$ & 15.6252 & 19.4137 & 22.5090 \\
\hline$\frac{\Delta B W}{B W_{0}}$ & $6.76 \%$ & $31.6 \%$ & $6.47 \%$ \\
\hline$P_{1}$ & 0.1447 & 0.1236 & 0.099 \\
\hline$P_{2}$ & 0.0892 & 0.0807 & 0.110 \\
\hline$\frac{\Delta P}{P_{0}}$ & $47.5 \%$ & $42.0 \%$ & $10.5 \%$ \\
\hline
\end{tabular}

Table 1: The detailed data of above spectrum in Fig. 5. The existence of $f$ can be inferred from a comparison of the first and third resonance. The peak with larger bandwidth has a higher peak power; comparing the first and second resonance, the relative difference in bandwidths of first resonance is only $6.7 \%$, whereas that in second resonance is $31.6 \%$, but the first one shows a even larger difference in peak power $(47.5 \%)$ than the second one $(42 \%)$; This uncoupling between relative peak power and bandwidth can only be explained with a non-zero $f$.

power should correspond with a large difference in peak bandwidth. However, we observe something different in the measured spectra shown in Table 1. In the first and third resonance, the peak with the larger bandwidth actually has a higher peak power. In addition, when comparing the first and the second resonance we also find a discrepancy: in the first resonance, the two peaks have a much smaller difference in bandwidth (6.7\%) than in the second resonance (31.6\%), but at the same time the first resonance has a larger difference in power $(47.5 \%)$ than the second resonance $(42 \%)$. We already explained that $\phi_{\mu}$ is very close to 0 , meaning that the difference in bandwidth can be very small. All of these phenomena reveal that, besides bandwidth, there must be another factor that introduces an asymmetry in order to explain the difference in peak power of a split resonance.

We can back this observation with t-CMT simulations in the circuit simulator Caphe $[21,22]$ by Luceda Photonics. When we simulate a ring resonator with backscattering, but without backcoupling in the directional coupler, we only get symmetrically split resonances. After introducing backcoupling into coupler model, we clearly observe the asymmetrically split resonances, as shown in Fig. 6. 


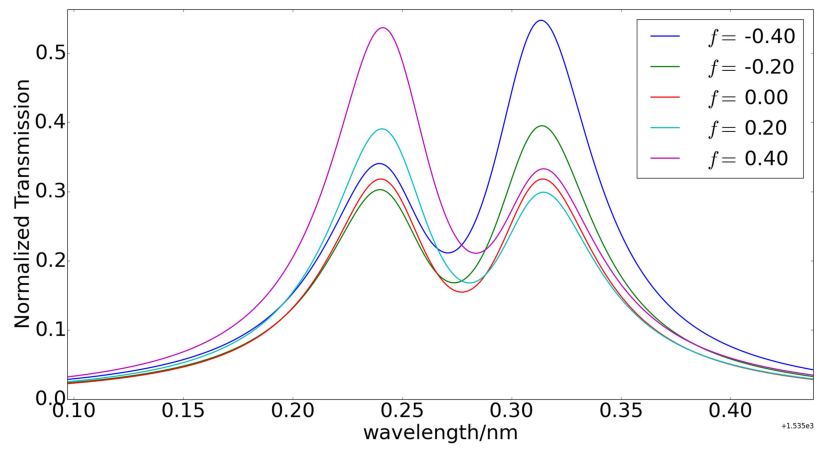

Figure 6: A sweep of different $f$ factor using circuit simulator Caphe. When $f=0$ and $\phi_{\mu}=0$, we get the red line, which is a symmetric split resonance; And when we increase $\mathrm{f}$, the degree of asymmetry increases.

\section{Individual contributions to backscattering}

A ring resonator consists of a circular waveguide and one or two directional couplers. Each component can potentially introduce unwanted reflection or scattering, contributing to the total backscattering in ring. We will now discuss these contributions in more detail.

\subsection{Sidewall Roughness-Induced Backscattering}

For the ring waveguide itself, roughness-induced backscattering is the only verified and detailed analyzed contribution to backscattering in the ring. Other potential contributions from the ring waveguide could include the transitions between a bend and a straight waveguide. In [23], the bend radius of ring resonator is chosen as large as $20 \mu \mathrm{m}$. At such large radii, the transition between bend and straight waveguide is almost perfect in silicon wire waveguides [24], which might explain why only roughness induced backscattering was observed. For sharper bend radii, $5 \mu \mathrm{m}$ or even smaller, the interface between bend and straight section can introduce additional reflections.

According to [16], roughness-induced backscattering can be considered as a statistical process, where the reflectivity's spectral characteristics (mean value, standard deviation and correlation length) depend on the waveguide length. Using optical frequency-domain reflectrometry (OFDR), Morichetti [16, 25] demonstrated a linear relationship between reflected power $R_{w}$ and waveguide length if the waveguide is short compared to the decay length of the propagation losses. For a ring resonator, the same technique clearly shows how a coherent addition of the reflections for each roundtrip in the ring increases the reflected power around resonance wavelength. This linear relationship is:

$$
R_{w}=r_{w}^{2}=H_{w} \times L_{w}
$$

Here, $H_{w}$ is a parameter dependent on waveguide dimension and sidewall quality.

\subsection{Coupler-Induced Backscattering}

In addition to the waveguide roughness, the directional couplers should also be considered as a source of backscattering. Ideally, a directional coupler shown in Fig. 1c does not have any reflection $r^{\prime}$ to the in port and backcoupling $k^{\prime}$ to the port adjacent to the in port, leaving only forward coupling $k$ and transmission $t$. But this is not always the case: the experimentally determined increase in loss in coupling sections [26] indicates that this reflection component can be non-negligible in microrings.

There can be two different types of backscattering associated to a directional coupler (Fig. 1d):

1. The existence of an adjacent waveguide is actually a perturbation to the refractive index of the original waveguide. So the beginning and end interface of a directional coupler behave like scatterers due to a too abrupt change in effective index. This scattering can couple to the backward propagating waveguide modes, i.e. an unwanted field backreflection $r$ to the input port and a backcoupling $k^{\prime}$ to the adjacent port. The more abrupt this transition from an isolated waveguide to a pair, the stronger we can expect the scattering to be. The abruptness increases for smaller bend radii, so we can expect a larger backscattering of the coupling sections in rings with a smaller bend radius.

2. For directional couplers with long (straight) coupling section, the power is exchanged back and forth between the two waveguides, increasing locally the field intensity at the gap walls, this is verified by FDTD simulation provided by Lumerical as illustrated in Fig. 7. Therefore, for the same waveguide length, there can be significantly higher backscattering by sidewall roughness compared to a single waveguide. We indicate the coupler roughness-induced backscattering as $r_{c}$. Similar to waveguides (equation (18)), the total reflected power $R_{c}=r_{c}^{2}$ has a form of:

$$
R_{c}=H_{c} \times L_{c}, \quad \text { where } H_{c}>H_{w}
$$

\subsection{Separation of Distributed and Lumped Backscattering}

In our t-CMT model for a ring resonator, we include both backscattering $r_{b s}$ and backcoupling $f$. The backscattering $r_{b s}$ consists of distributed backscattering caused by waveguide roughness $\left(r_{w}\right)$, whose power reflectivity has a linear dependence on ring length $L$, and lumped reflection introduced by couplers $\left(r^{\prime}\right.$ and $\left.r_{c}\right)$, whose power reflectivity should be independent on ring length, but dependent on couplers parameters, for instance, gap and coupling length. Based on this knowledge, assuming a low average total reflectivity during one roundtrip, only first order reflection contributions are taken into account. When the ring length $L<<\frac{1}{2 \alpha_{\text {loss }}}$ we propose for low-field reflectivity $r_{b s}$ a simple linear approximation of power reflectivity $R_{b s}=r_{b s}^{2}$ :

$$
R_{b s}=H_{0} L+C_{0}
$$




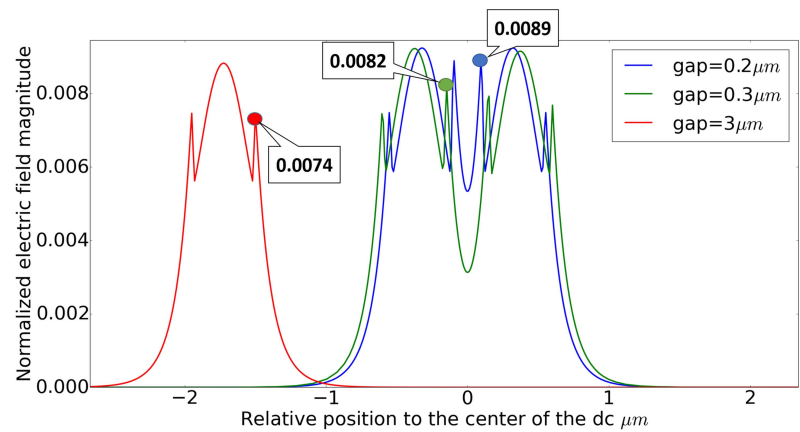

Figure 7: Three mode profiles extracted from commercial FDTD simulator provided by Lumerical. Blue and green indicate two directional couplers with a gap of $200 \mathrm{~nm}$ and $300 \mathrm{~nm}$ respectively, while red one refers to a single mode waveguide. Clearly, the electric field magnitude at the sidewall of a directional coupler is stronger than that of a waveguide.

$H_{0} L_{0}$ refers to the distributed backscattering caused by waveguide roughness and $C_{0}$ covers the contribution from the couplers.

\section{Experiment results}

A set of rounded rectangular add-drop microrings (fixed $6.5 \mu \mathrm{m}$ coupling length and $4.5 \mu \mathrm{m}$ bend radius) with 7 different total ring lengths $(150-1000 \mu \mathrm{m})$ and 6 different coupling gaps (150-400 nm) were measured in order to verify the model proposed in equation (20). With this, we intend to verify that the sources of backscattering include circular waveguide roughness as well as directional couplers. We analyzed all resonances between $1520 \mathrm{~nm}$ and $1560 \mathrm{~nm}$, using a $1 \mathrm{pm}$ resolution wavelength scan with a continuous-wave tunable laser and power meter in a vertical coupling setup. The devices were designed with the IPKISS framework [27] and fabricated at IMEC in a passive silicon photonics technology [28]. The waveguide dimensions are $450 \mathrm{~nm} \times 220 \mathrm{~nm}$, embedded in oxide and excited with TE polarization using grating fiber couplers.

\subsection{Fitting}

First of all, we verify our model by testing its capability to fit all the peaks of the different ring resonators. Equation (10) is implemented into our Python modeling code and the parameters are fit to the resonance spectrum of the drop port using a least-square algorithm. With small modifications, the same procedure can be applied to the pass port spectrum. The free parameters in frequency domain are $\left[P_{p}, \omega_{0},\left.B W_{0}\right|_{\omega}, \mu_{0}, f\right.$, $\left.\phi_{\mu}\right]$, or similarly in wavelength domain $\left[P_{p}, \lambda_{0},\left.B W_{0}\right|_{\lambda}, \mu_{0}\right.$, $f, \phi_{\mu}$ ], which correspond to [peak power, central frequency, $3 \mathrm{~dB}$ bandwidth, amplitude of backscattering, backcoupling factor, phase of backscattering], respectively. The values of $P_{p}, \omega_{0}$ and $\left.B W_{0}\right|_{\omega}$ correspond to the resonance of the ideal ring as if there was no backscattering present, while the other parameters $\mu_{0}, f$, and $\phi_{\mu}$ describe the perturbation due to the backscattering.

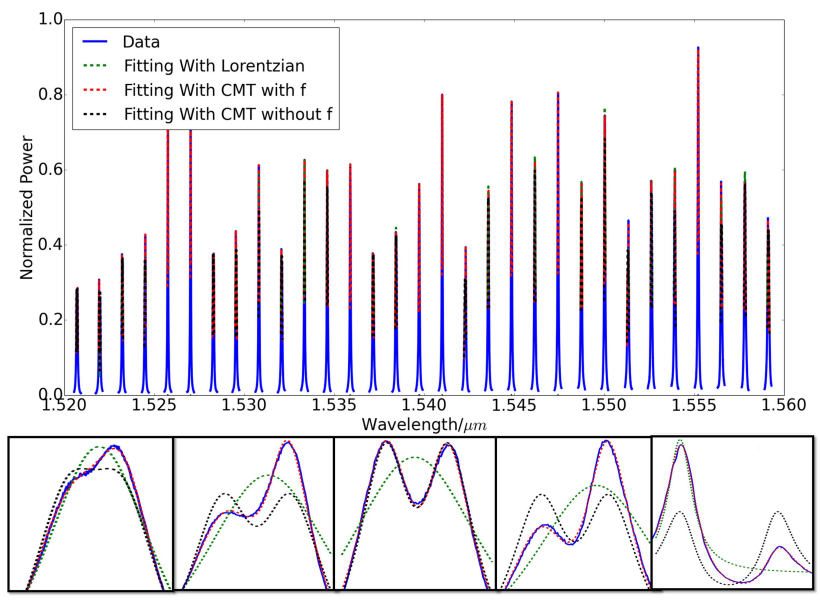

Figure 8: Comparison of fitting results with 3 different models. Measured data is shown in blue solid line; in green dashed line we fit with traditional Lorentzian shape. The black dashed line gives the fitting with our t-CMT model but without backcoupling factor $f$. Clearly, only symmetrically split resonances are correctly handled. The fitting results of our improved t-CMT model with $f$ is shown in red dashed line, which closely matches the measurements.

Figure 8 gives some examples of 3 different ring models. The green line is the result of a traditional Lorentzian method. It fails to fit every resonance with even a small amount of peak splitting. The black curve fits the resonances with a t-CMT model with backscattering, but without backcoupling $(f=0)$. Only symmetrically split resonances can be represented by this model, and it performs poorly for asymmetric peaks. Our improved t-CMT model with a nonzero backcoupling factor $f$ is plot in a red dashed line. It accurately fits both non-split, symmetrically split and asymmetrically split resonances.

Figure 9 plots the $f$-factor for a typical example of our measured rings. The amplitude of $f$ factor varies from 0 to 0.4 , which means that there can be as much as $40 \%$ of the

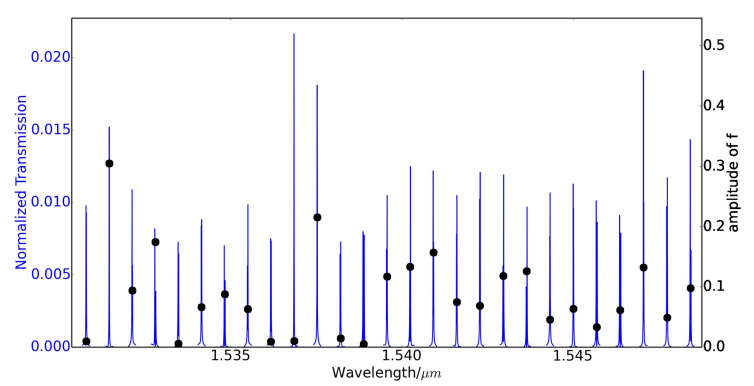

Figure 9: A measured spectrum with fitted amplitude of $f$ factor at each resonance. Similar in other measured spectra, the amplitude of $f$ is in the range of 0 to 0.4 . 
cross-coupled field coupled back to the adjacent port of in port. Such a large value explains the significant difference in peak power in some split resonances.

\subsection{Extracting the Backscattering}

With an accurate peak-fitting model we can now look deeper into the actual backscattering in SOI microrings. First of all, we will mathematically extract the strength of the backscattering from measured spectra. By analyzing the transmission spectra at the drop port and the add port, we can extract the field backreflectivity $r_{b s}$ for each individual resonance. As the $r_{b s}$ variation with wavelength has a strong stochastic component, we look at the mean and standard deviation for $r_{b s}$ over the different resonances within the transmission spectrum of a single ring. For resonances that are visibly split, we calculate $r_{b s}$ as:

$$
R_{b s}=r_{b s}^{2}=\mu_{0}^{2} \frac{n_{g} L}{c}=\mu_{0}^{2} \frac{\lambda_{0}^{2}}{c \times F S R_{\lambda}},
$$

where $F S R_{\lambda}$ is the free spectral range in wavelength domain, $\lambda_{0}$ is the central wavelength of the fitted resonance. All of these parameters, $\lambda_{0}, F S R_{\lambda}$ and $\mu_{0}$ can be directly extracted from the fit of the peak. From equations (14) and (15) we could get equation (22):

$$
\Delta \omega_{b s}=\omega_{1}-\omega_{2}=2 \mu_{0} \cos \frac{\phi_{\mu}}{2}=\frac{2 \pi c}{\lambda_{0}^{2}} \Delta \lambda_{b s}
$$

And as discussed in section 2 , the $\phi_{\mu}$ is always very close to 0 , we further simply it as equation:

$$
\mu_{0}=\frac{\pi c}{\lambda_{0}^{2}} \Delta \lambda_{b s}
$$

Now in combination with equation (7), equation (24) can be generated.

$$
\frac{\Delta \lambda_{b s}}{B W_{\lambda}}=\frac{\mathcal{F} r_{b s}}{\pi},
$$

where $\Delta \lambda_{b s}$ refers to the wavelength spacing between the two peaks of a split resonance. $\mathcal{F}=\frac{F S R_{\lambda}}{B W_{\lambda}}$ is the finesse of the ring, where $\mathcal{F} / 2 \pi$ is the number of roundtrips light makes during the cavity lifetime. This equation formulates mathematically how at resonance the backreflection during one roundtrip is coherently added up for each of the $\mathcal{F} / 2 \pi$ roundtrips.

However, for non-split peaks, the fitted parameter $\mu_{0}$ is not always reliable, as the envelope shape of the combined peak is less unambiguously dependent on the exact properties of the individual resonance peaks: there are often multiple solutions for the resonance modes $\alpha_{c w}$ and $\alpha_{c c w}$. Instead, we can use the relative peak intensity at the add and drop port to calculate $r_{b s}$. After some transformations of equations (10) and (12), we get

$$
\frac{P_{A}}{P_{D}}=\frac{\left(\frac{\Delta \lambda_{b s}}{B W_{\lambda}}\right)^{2}}{1+\left(\frac{\Delta \lambda_{b s}}{B W_{\lambda}}\right)^{2}}=\frac{\left(\frac{\mathcal{F} r_{b s}}{\pi}\right)^{2}}{1+\left(\frac{\mathcal{F} r_{b s}}{\pi}\right)^{2}}
$$

Similar to equation (24), the dependency on $\mathcal{F} r_{b} s$ reflects the coherent addition of reflection per roundtrip at resonance.

\subsection{Separating Distributed and Lumped Backscattering}

In equation (20) we separated the total backscattering into contributions by lumped scatterers $\left(C_{0}\right)$ and contributions by distributed scatterers $\left(H_{0}\right)$, like sidewall roughness. $H_{0}$ depends on the electric field strengths at the sidewalls and the quality of sidewall roughness. A lower value of $H_{0}$ can be obtained by using a better etch process, broader waveguides, or by using the TM polarization [25,29].

We applied our model and extraction procedures to rings with different roundtrip length and coupling gaps. The propagation losses in the measured waveguides are of the order of $<2 \mathrm{~dB} / \mathrm{cm}$ [8]. This makes, even if the additional losses in the coupling sections of small gap resonators are considered, the linear approximation in equation (20) a valid assumption.

We plot the power reflectivity $R_{b s}$ in Fig. 10, which confirms that the highest power reflection $R_{b s}$ per roundtrip is of the order of 0.01 , and therefore higher order reflection contributions can indeed be safely neglected in equation (20).

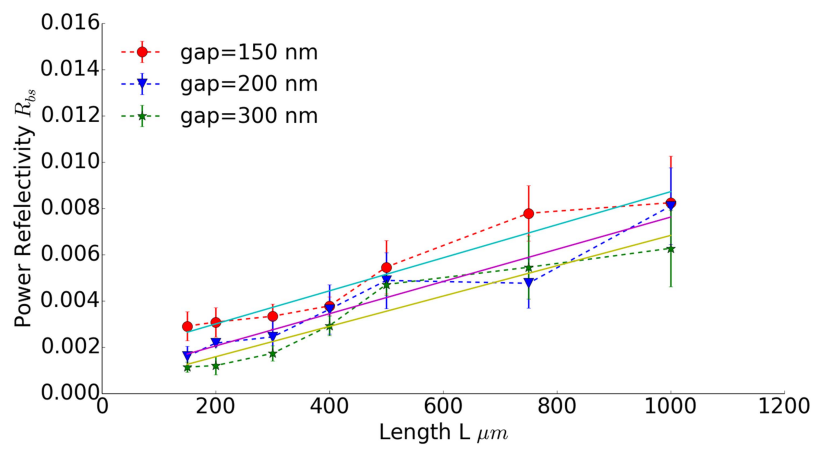

Figure 10: Power backscattering per roundtrip for rings with different roundtrip length and different directional coupler gap. We see a linear increase of the backscattering for longer rings, which corresponds to distributed scattering $H_{0}$. We also see a decrease in backscattering for larger coupler gap, corresponding to lumped scattering $C_{0}$ in the directional couplers.

We clearly see that the reflected power increases linearly with the ring length, confirming the length-dependent model from equation (20). We also see that for a larger coupler gap, the curve shifts down. This confirms that the directional coupler plays an important role in the lumped scattering contribution $C_{0}$, and that smaller gaps will lead to stronger discontinuities and backscattering. 


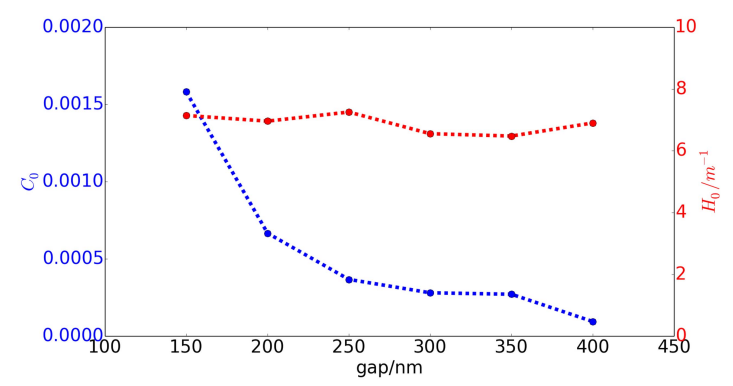

Figure 11: Fitted parameters $H_{0}$ and $C_{0}$ versus coupler gap. $H_{0}$ remains constant for different gaps, as it only depends on the circular waveguide roughness. The value is well matched with formerly reported value. $C_{0}$ decreases for increasing gap, as the larger gap is, the smaller influence of directional coupler will be.

We see this confirmed in Fig. 11 where dependence of $C_{0}$ and $H_{0}$ on the coupler gap is plotted. For all gaps, $H_{0}$ is quite stable at the value around $6-8 \mathrm{~m}^{-1}$, which corresponds well with the value reported in [16]. As $H_{0}$ represents the backscattering caused by sidewall roughness, we indeed expect it to be independent of the gap. In terms of $C_{0}$, it is clearly shown in the same figure that rings with larger gap have smaller $C_{0}$, meaning less backscattering is induced by the couplers.

\subsection{Analysis of the Coupler-Induced Backscattering}

$C_{0}$ is more complicated to understand than $H_{0}$. For simplicity, let us assume that the lumped contributions to backscattering solely originate in the directional couplers (actually, the bend/straight transition might introduce extra lumped reflections. This could be considered as a weak incremental to the $C_{0}$, and this incremental should be independent on couplers parameters). The bend radius, the gap and the coupling length have been experimentally proven to play a role. We investigated the effect of the couplers by measuring another set of rings with a fixed coupler gap $(200 \mathrm{~nm}), 7$ different ring roundtrip lengths (150-1000 $\mu \mathrm{m})$, and 6 different coupling lengths $(5-15 \mu \mathrm{m})$.

Figure 12 again plots $R_{b s}$ as function of ring length, but now for directional couplers with 3 different coupler lengths. The curves with the longer coupling lengths $L_{c}$ show a higher backscattering. Figure 13 quantifies how $C_{0}$ increases with coupling length $L_{c} . H_{0}$ also shows a slight increase, but less pronounced, and still within the range reported in [16]. The change in $H_{0}$ can also be due to a secondary effect: longer coupler lengths increase the ring linewidth, which affects the quality of the fit.

All of the data shown above tell us that the coupler sections indeed introduce considerable reflections, and the strength of that reflection depends on both gap and coupling length.

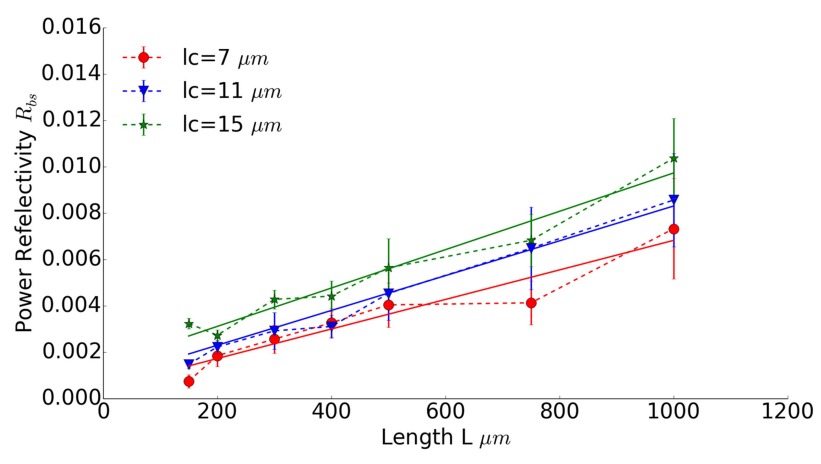

Figure 12: Power backscattering as function of ring roundtrip length for different coupling lengths $L_{c}$. The backscattering increases with larger roundtrip lengths, and with larger coupling length.

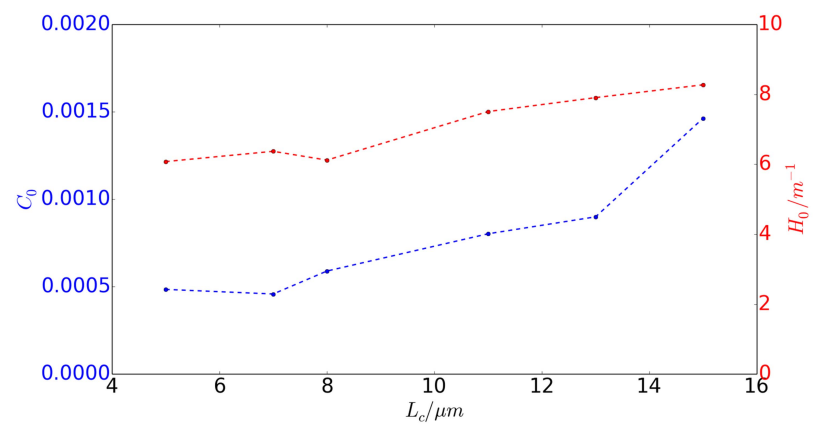

Figure 13: Fitted backscattering contributions $H_{0}$ and $C_{0}$ for different coupling lengths $L_{c}$. $C_{0}$ increases with increasing coupling length, indicating more backscattering caused by directional couplers. $H_{0}$ remains in the expected range of $6-8 m^{-1}$

\section{Wavelength-dependent Model for Full-Spectrum Fitting}

With the model discussed in the previous section, we could accurately fit every individual resonance separately.

However, in reality, there should be a constant set of parameters for the ring circuit instead of set of parameters for each of the resonances. By claiming that each resonance has different parameters, i.e. backcoupling and backscattering, we could explain the differences between resonances in a single spectrum. However, the question still remains how to physically explain the differences between the parameters of different resonances.

When we go back to our former analysis, we remind ourselves that there are two individual contributions: roughnessinduced backscattering and directional couplers. It has already been reported in [16] that the roughness-induced backscattering exhibits a degree of randomness, which might be a reason for the different shapes of resonances in a single spectrum. However, our analysis and measurement results in previous sections reveal that the strength of backscattering only influences the spectral separation of 
the two split peaks. In other words, backscattering only influences the resonances' wavelengths (frequency) and their bandwidths. The asymmetry and large differences in bandwidth in each split-resonance can not be explained by the randomness in backscattering.

When we consider the layout of the system, we see that for an add-drop filter, we have two couplers, so four scatterers in total. These four scatterers plus backscattering will form a complicated multi-cavity system in the ring circuit, which will show a certain wavelength dependency. In order to verify this, we use the circuit simulator Caphe with a detailed model for the ring circuit to fit our measured data. The fitting procedure used is differential evolution [30]. In this model, a basic directional coupler contains two waveguides with different effective index simulated by FIMMWAVE, each supporting a supermode. We add extra scatterers at the input and output sides of a directional coupler to include their parasitic reflection and backcoupling. Figure

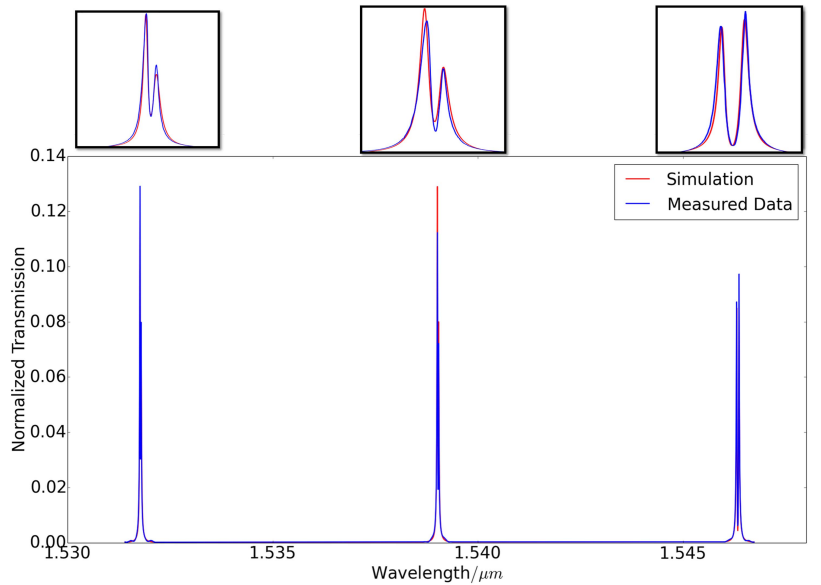

Figure 14: Fitting of a narrow span of a 75um long ring; here we use our circuit simulator CAPHE to fit a ring circuit, or in other words, the complete spectrum instead of fitting individual resonance one by one

14 and 15 show the fitting of two relatively narrow spans. Due to the limited number of resonances in the spectrum, the randomness of the backscattering does not play a significant role. Even if in our model we use a constant value for backscattering, we have still have a relatively good match between simulation and measured data, as well as with the previously fitted and simulated values. For example, the power coupling ratio is in the order of $1 \times 10^{-3}$, similar to the value simulated by Lumerical which is $3.6 \times 10^{-3}$. The fitted value of backcoupling of the directional coupler is in the order of $10^{-5}$, in good correspondence with the previously reported $f$-factor for the electric field, which is from 0 to 0.3 , so from 0 to 0.09 for power.

Figure 16 shows the fitting result of a longer ring, with more than 10 peaks. Due to the large number of resonances here, the randomness of the backscattering can become quite influential and will have an impact on the fitting quality, as for this fitting procedure we use a constant amplitude value of the backscattering in our circuit model. As expected, the

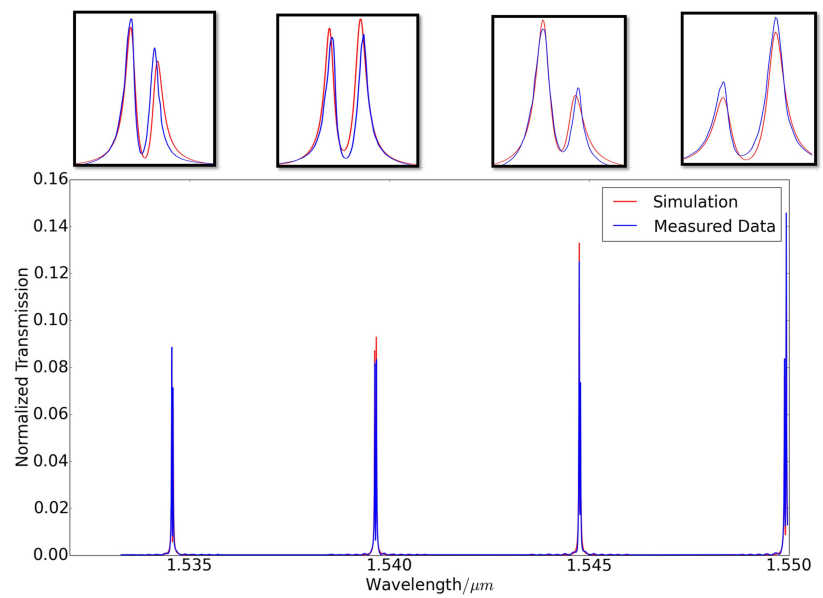

Figure 15: Fitting of a narrow span of a 100um long ring, shows the reproducibility of our circuit fitting model

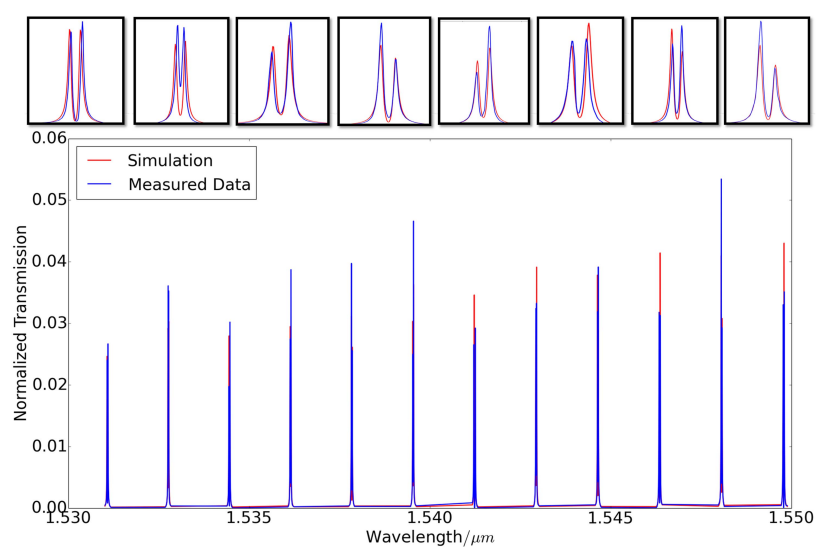

Figure 16: Fitting of a narrow span of a 300um long ring; We expect larger mismatch due to the randomness of roughness induced backscattering, which is not included in our model. However, there is still a good match and it clearly shows the randomness in split resonances caused by multi-scatters in the ring circuit

fitting mismatch increases, which can actually be considered as a measure for randomness of the distributed backscattering. Still, we can extract trends from this fitting.

In summary, we identify two reasons in order to explain why individual resonances in the same ring can be quite different, not only in split ratio, but also in asymmetry and bandwidths. The first one is due to the previously reported randomness in waveguide-roughness-induced backscattering, and this will affect the split ratio of the resonances. The second contribution is from the complicated directional couplers, which contain not only lumped multi-scatterers that introduce extra reflection and backcoupling but also distributed backscattering due to the sidewall roughness of the extra coupling length. 


\section{Methods to suppress resonance splitting}

Understanding the origin of the resonance peak splitting, we can now suggest a number of techniques to reduce or avoid resonance splitting. Generally, the methods to suppress resonance splitting can be classified into four categories:

1. Improving the lithography and etching technology to reduce the sidewall roughness [25].

2. Using TM polarisation or rib waveguides so that the sidewall sees weaker electric field [25,29].

3. Lowering the $Q$ factor of the ring, i.e. strengthening the coupling coefficient of the directional coupler(s). Among the 6 measured sets of rings with gaps from $0.15 \mu \mathrm{m}$ to $0.4 \mu \mathrm{m}$, there is a clear observable trend that resonance splitting is very rare in rings with gap of $0.15 \mu \mathrm{m}$. While on the other hand, rings with a gap larger than $0.25 \mu \mathrm{m}$, almost invariably exhibit severe resonance splitting due to a narrower bandwidth/higher $Q$ factor, as illustrated in Fig. 3.

4. By design optimization: we could tailor either the backscattering $r_{b s}$ inside the ring waveguide or the backcoupling ( $f$ factor) of the whole ring circuit. The former method relies on an intentional reflector inside the ring, whose reflectivity as well as phase could be tuned, to compensate the parasitic backscattering $r_{b s}$. As a consequence, the total ring circuit can be made to suffer no backscattering at all. The second method tunes the $f$ factor to be 1 , so that the coupling from the input wave contributes equally to $\mathrm{CW}$ and $\mathrm{CCW}$ modes. As in equation (10) shows, when $f=1$, one of the peaks of a split resonance disappears, with only one resonance left. In such a case, the parasitic backscattering as well as the resonance splitting still occurs, but one peak is suppressed at the output.

\section{Conclusions}

This paper explains quantitatively the wide variation of resonance splitting in ring resonators. For this, we performed a comprehensive and in-depth analysis of backscattering, one of the most severe as well as frequently observable problems in silicon microring resonators, based on measurements of abundant devices fabricated in mature CMOS technology. This quantitative analysis demonstrated the high probability of resonance splitting and the dominance of asymmetric ones in those resonance splitting. It also illustrated the problem of wide variations in resonance splitting of a single ring's spectrum is observed and proposed.

In order to explain and model the resonance splitting ( especially the asymmetric splitting, which was hitherto impossible to model accurately ), we developed a model based on temporal coupled-mode theory, which incorporates the parasitic processes of backscattering (distributed and lumped) as well as backcoupling in the coupler sections. We get a good fitting in numerous ring spectra with both single and split resonances, symmetric or asymmetric. We also theoretically and experimentally proved that the origin of the asymmetry in split resonances is the backcoupling in the directional couplers. This model enabled us to get a deeper understanding of the mechanisms that controbute to backscattering in SOI microrings. The measurement results confirmed our hypothesis that backscattering is not only caused by sidewall roughness but also by the directional couplers. We also characterized in more detail the relative influence of coupler's gap and coupling length.

After successfully characterizing the splitting in a single ring resonance, we improved our behavioral model to explain the wide variations in resonance splitting within one transmission spectrum. In this improved model, the directional coupler is modeled in more detail by decomposing it into multiple sections: two scatterers at the beginning and end section that can introduce lumped reflection and backcoupling, and backscattering 'waveguides' corresponding to the two supermodes in the directional coupler. The satisfying fitting results confirm that the very diverse cases of resonance splitting within one spectrum is indeed caused by the complicated multi-cavity system.

These models and characterization results will be invaluable to improve the performance of silicon microring resonators and suppress the resonance splitting. The model can also help to use the parasitic backscattering and resonance splitting for more useful purpose, such as reflectors for a dual wavelength laser or novel sensing schemes.

Key words: Optical filters, optical waveguides, silicon photonics, integrated optics, curve fitting

\section{References}

[1] P. De Heyn, J. De Coster, P. Verheyen, G. Lepage, M. Pantouvaki, P. Absil, W. Bogaerts, J. Van Campenhout, and D. Van Thourhout, Lightwave Technology, Journal of 31(16), 2785-2792 (2013).

[2] K. De Vos, I. Bartolozzi, E. Schacht, P. Bienstman, and R. Baets, Optics express 15, 7610-7615 (2007).

[3] K. Vandoorne, P. Mechet, T. Van Vaerenbergh, M. Fiers, G. Morthier, D. Verstraeten, B. Schrauwen, J. Dambre, and P. Bienstman, Nature communications 5 (2014).

[4] S. Gulde, A. Jebali, and N. Moll, Optics express 13, 95029515 (2005).

[5] Q. Xu, V. R. Almeida, and M. Lipson, Optics letters 30, 2733-2735 (2005).

[6] H. Shao, H. Yu, X. Jiang, J. Yang, and G. Roelkens 4, 47-48 (2014).

[7] T. Chu, N. Fujioka, and M. Ishizaka, Optics express 17, 14063-14068 (2009).

[8] W. Bogaerts, P. De Heyn, T. Van Vaerenbergh, K. De Vos, S. Kumar Selvaraja, T. Claes, P. Dumon, P. Bienstman, D. Van Thourhout, and R. Baets, Laser Photonics Reviews 6, 47-73 (2012).

[9] J. Heebner, R. Grover, and T. Ibrahim, Optical Microresonators: Theory, Fabrication and Applications, 1st edition, Springer Series in Optical Sciences (Springer, Germany, 2008).

[10] B. E. Little, J. P. Laine, and S. T. Chu, Optics letters 22, 4-6 (1997).

[11] S. Werquin, S. Verstuyft, and P. Bienstman, Optics express 21(14), 16955-16963 (2013). 
[12] M. Moresco, M. Romagnoli, S. Boscolo, M. Midrio, M. Cherchi, E. S. Hosseini, D. Coolbaugh, M. R. Watts, and B. Dutt, Optics express 21(5), 5391-400 (2013).

[13] Z. Zhang, M. Dainese, L. Wosinski, and M. Qiu, Optics express 16, 4621-4630 (2008).

[14] G. C. Ballesteros, J. Matres, J. Mart, and C. J. Oton, Optics Express 19(25), 24980-24985 (2011).

[15] S. Tallur and S. a. Bhave, Opt. Express 21(23), 2778027788 (2013).

[16] F. Morichetti, A. Canciamilla, and A. Melloni, Optics letters 35, 1777-1779 (2010).

[17] J. Čtyroký, I. Richter, and M. Šior, Optical and Quantum Electronics 38(9-11), 781-797 (2007).

[18] B. Little, S. Chu, H. Haus, J. Foresi, and J. P. Laine, Journal of Lightwave Technology 15(6), 998-1005 (1997).

[19] D. Melati, A. Melloni, and F. Morichetti, Advances in Optics and Photonics 6(2), 156-224 (2014).

[20] M. A. Popović, Theory and Design of High-Index-Contrast Microphotonic Circuits, PhD thesis, 2008.

[21] M. Fiers, T. V. Vaerenbergh, K. Caluwaerts, D. V. Ginste, B. Schrauwen, J. Dambre, and P. Bienstman, J. Opt. Soc. Am. B 29(5), 896-900 (2012).

[22] T. Van Vaerenbergh, M. Fiers, J. Dambre, and P. Bienstman, Optical and Quantum Electronics pp. 1-6 (2015).

[23] F. Morichetti, a. Canciamilla, M. Martinelli, a. Samarelli, R. M. De La Rue, M. Sorel, and a. Melloni, Applied Physics Letters 96(May), 13-15 (2010).

[24] W. Bogaerts, P. Dumon, D. V. Thourhout, D. Taillaert, P. Jaenen, J. Wouters, S. Beckx, V. Wiaux, and R. G. Baets, Selected Topics in Quantum Electronics, IEEE Journal of 12(6), 1394-1401 (2006).

[25] F. Morichetti, A. Canciamilla, C. Ferrari, M. Torregiani, A. Melloni, and M. Martinelli, Physical review letters 104(3), 033902 (2010).

[26] F. Xia, L. Sekaric, and Y. a. Vlasov, Optics express 14(9), 3872-86 (2006).

[27] M. Fiers, E. Lambert, S. Pathak, B. Maes, P. Bienstman, W. Bogaerts, and P. Dumon, Journal of Computational Science 4(5), 313-324 (2013).

[28] S. K. Selvaraja, P. Jaenen, W. Bogaerts, D. Van Thourhout, P. Dumon, and R. Baets, Lightwave Technology, Journal of 27(18), 4076-4083 (2009).

[29] P. J. De Heyn, B. Kuyken, D. Vermeulen, W. Bogaerts, and D. Van Thourhout, High-performance low-loss siliconon-insulator microring resonators using tm-polarized light, in: Optical Fiber Communication Conference, (2011), p. OThV2.

[30] P. Rocca, G. Oliveri, and A. Massa, Antennas and Propagation Magazine, IEEE 53(1), 38-49 (2011).

Ang $\mathbf{L i}$ is a full time Ph.D student in the Photonics Research Group at Ghent University-IMEC. He graduated from an Erasmus Mundus Master Program-MAPNET (Master On Photonics Network) in 2013, with M.Sc. degrees from Technical University Berlin and Scuola Superiore Sant'anna. Before that, he got a B.Sc. degree from microelectronics department of Fudan University, Shanghai, China in 2011. He has a strong interest in design, simulation and characterisation of integrated photonics devices.
Thomas Van Vaerenbergh was born in Ghent, Belgium, 1987 He received the M.Sc. degree in applied physics from Ghent University, Ghent, Belgium, in 2008. Currently, Thomas is with the Photonics Research Group, Department of Information Technology and HP Labs, Palo Alto, California, United States. His research topic is photonic reservoir computing. His research interests include excitability in photonic components and the modeling of nonlinear photonic cavity circuits.

Peter De Heyn received the masters degree in photonics engineering from Ghent University and Vrije Universiteit Brussel (VUB), Belgium in 2009. He is now working towards a PhD degree in the Photonics Research Group of Ghent University and IMEC, Belgium. He was a 6 months visiting researcher at HHI Fraunhofer Institute, Berlin, Germany in 2011. His interest is optical interconnects on silicon photonics including ring resonators for Iter applications, heterogeneous III-V on $\mathrm{SOI}$ integration, high-performance III-V and germanium photodiodes.

Peter Bienstman was born in Ghent, Belgium, 1974. He received the M.Sc. degree in electrical engineering and the Ph.D. degree from Ghent University, Ghent, in 1997 and 2001, respectively. Currently he is an associate professor with the Department of Information Technology, Ghent University. During 2001-2002, he spent a year in the Joannopoulos research group at MIT. His research interests include several applications of nanophotonics (biosensors and photonic information processing) as well as nanophotonics modeling. He has published over 50 papers and holds several patents. He is a member of the IEEE Photonics Society. He has been awarded the European Research Council starting grant for the Narescoproject: Novel paradigms for massively parallel nanophotonic information processing.

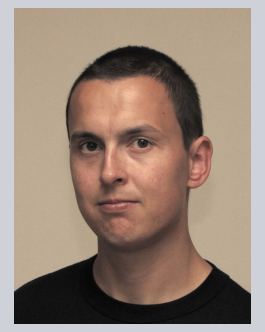

Wim Bogaerts is professor in the Photonics Research Group at Ghent University - imec. He received his $\mathrm{PhD}$ in the modelling, design and fabrication of silicon nanophotonic components at Ghent University in 2004. During this work, he started the first silicon photonics process on imecs $200 \mathrm{~mm}$ pilot line, which formed the basis of the multi-project-wafer service ePIXfab. Wims current research focuses on the design challenges for silicon photonics: Large-scale integration, circuit complexity, tolerances, parasitics and multiphysics, and their use in reconfigurable photonic circuits. In June 2014, Wim co-founded Luceda Photonics, a spin-off company of Ghent University, IMEC and the University of Brussels (VUB). Luceda Photonics develops unique software solutions for silicon photonics design, using the IPKISS design framework. Wim has a strong interest in telecommunications, information technology and applied sciences. He is a member of IEEE, Optical Society of America (OSA) and SPIE. 